\title{
Projecting future HFC-23 emissions
}

\author{
B. R. Miller ${ }^{1,2}$ and L. J. M. Kuijpers ${ }^{3}$ \\ ${ }^{1}$ Cooperative Institute for Research in Environmental Sciences, University of Colorado, Boulder, Colorado, USA \\ ${ }^{2}$ Global Monitoring Division, ESRL, NOAA, Boulder, Colorado, USA \\ ${ }^{3}$ Eindhoven Centre for Sustainability, Technical University Eindhoven, Eindhoven, Netherlands
}

Received: 11 July 2011 - Published in Atmos. Chem. Phys. Discuss.: 16 August 2011

Revised: 6 November 2011 - Accepted: 7 December 2011 - Published: 21 December 2011

\begin{abstract}
A Reference Case (RC) scenario for emissions of HFC-23 from co-production during HCFC-22 manufacture over the next 25 years is presented. Offered as a template rather than a prediction, this model projects current production practices and existing abatement frameworks to yield insights into how atmospheric composition and radiative forcing might change with and without additional efforts to constrain HFC-23 emissions.

Assuming that no additional abatement measures are implemented, emissions for year 2035 in this Reference Case

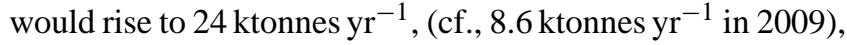
the atmospheric abundance of HFC-23 would rise to $50 \mathrm{ppt}$, which is a $121 \%$ increase over the 2009 observed abundance, and HFC-23 would be expected to contribute a radiative forcing of $9 \mathrm{~mW} \mathrm{~m}^{-2}$ (cf., $4 \mathrm{~mW} \mathrm{~m}^{-2}$ in 2009). Under such a scenario, the HFC-23 emission growth rate would be a con-

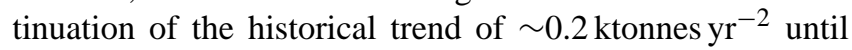
2030, after which the growth is projected to quadruple as the Montreal Protocol phase-out of HCFC production for dispersive use concludes and HFC-23 thermal decomposition in the projects of the Clean Development Mechanism (CDM) comes to a scheduled end while growth in the production of HCFC-22 for feedstock use continues to climb with projected GDP growth.

Two opposite variations regarding the future renewal of CDM projects are examined for their impact on projected emissions and abundance, relative to the Reference Case scenario.
\end{abstract}

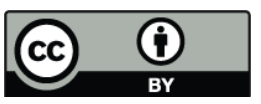

Correspondence to: B. R. Miller (ben.r.miller@noaa.gov)

\section{Introduction}

Hydrofluorocarbon-23 (HFC-23, also known as $\mathrm{CHF}_{3}$, trifluoromethane and fluoroform) is a long-lived (atmospheric lifetime $\sim 270$ years, WMO 2010) and potent greenhouse gas (GHG) with a global warming potential (GWP) of 14800 for a 100-year horizon (Forster et al., 2007). As an unintentional by-product of HCFC-22 manufacture at about a $2-3 \%$ yield, HFC- 23 is largely considered a waste gas that historically was, and still often is, vented directly to the atmosphere. Due to the fact that it finds little industrial use, HFC-23 is often overlooked (Velders et al., 2009) or relegated to a relatively trivial role (van Vuuren et al., 2010) in projections of climate change. However, the valuable fluoropolymers produced from HCFC-22 have become ubiquitous in the modern automobile, textile and construction material industries. While the production of HCFC-22 for dispersive uses is being phased out under the Montreal Protocol, production for use as feedstock in fluoropolymer manufacture is unrestricted. Of the major long-lived greenhouse gases (LLGHGs) discussed in the AR4 Report (IPCC, 2007), contributions to radiative forcing were dominated by four compounds $\left(\mathrm{CO}_{2}, \mathrm{CH}_{4}, \mathrm{CFC}-12\right.$ and $\left.\mathrm{N}_{2} \mathrm{O}\right)$. The combined contribution of the other fourteen considered species (including other CFCs, HCFCs, HFCs, PFCs, chlorocarbons and SF6) amounted to only $6 \%$ to the total $2.63 \mathrm{~W} \mathrm{~m}^{-2}$ in 2005 for all LLGHGs. However, the emission scenario proposed by Velders et al. (2009) suggests that the proportion of total radiative forcing from HFCs relative to that of $\mathrm{CO}_{2}$ is likely to increase in the coming decades. As these authors state, "The potentially large contribution of HFC emissions to future climate forcing in the coming decades has attracted the attention of policymakers seeking climate protection". Unlike many GHG sources that emit to the atmosphere over large spatial and temporal scales (e.g., methane from landfills and nitrous oxide from fertilization of agricultural fields), HFC-23 is produced within the confines of

Published by Copernicus Publications on behalf of the European Geosciences Union. 
a relatively small number of manufacturing facilities, where it may be destroyed efficiently through high temperature incineration. The cost of incineration varies regionally, and also depends on whether it is an installation in a new or existing facility. Estimates given by the EPA (2006) indicate that installation costs range \$3-4 million, with annual operating costs of order $\$ 200-300$ thousand per year. Installations capable for all or most of a plant's HFC-23 production have been voluntarily installed already throughout $\mathrm{Eu}-$ rope and the US, suggesting that industry is embracing this mitigation strategy. Thus, the anticipated inseparability of industrial growth and HCFC-22 manufacture suggests that mitigation of HFC-23 co-production will be an important focus for policymakers seeking cost effective means of reducing GHG emissions for some time to come.

As one of the six classes of greenhouse gases in the Kyoto Protocol, annual HFC emission reporting has been an obligation of Annex I countries to the United Nations Framework Convention on Climate Change (UNFCCC, 2010) since 1990. Emissions of HFC-23 from these developed countries have been greatly reduced since that time, largely due to voluntary abatement measures that include process optimization, whereby the co-production yield is reduced, and by incineration. Beginning in 2003, certain facilities in developing countries were approved as projects of the Clean Development Mechanism (CDM) under the Kyoto Protocol and were eligible to produce Certified Emission Reduction (CER) credits for the destruction of their co-produced HFC23. By 2008, incineration by the CDM projects destroyed about half of the annual HFC-23 production in the developing world (Miller et al., 2010). At a time when HCFC22 production was rising rapidly in the developing countries, incineration within these CDM projects was largely responsible for reversing the previously positive growth trend in global HFC-23 emissions and producing an emission rate of

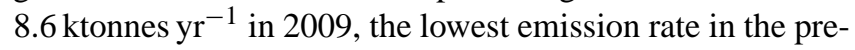
vious fifteen years. By the year 2009, eighteen out of the nineteen approved projects had commenced thermal decomposition of the HFC-23 produced at their facilities. As these facilities demonstrated success in destroying virtually all of their production (Miller et al., 2010), and no additional developing country facilities currently meet requirements for project registration under current CDM policy (UNFCCC, 2010), we anticipate that the amount of HFC-23 produced in the developing world that will be abated by these projects in the future will not increase significantly. This paper examines the future balance of production and destruction anticipated for HFC-23 by detailed analysis of these controlling factors.

The bottom-up HFC-23 emission history derived in Miller et al. (2010) decomposed the global picture of HFC-23 emissions into components that readily lend themselves to projection. Explicitly defined quantities of HFC-23 production and incineration along with HCFC-22 production within the developing world, combined with reliable emission reporting by the developed world to the UNFCCC, lead to a global bottom-up emission history that agreed within stated uncertainties with a top-down emission history derived from inverse modeling of atmospheric observations. Here, with simple assumptions, we extend these bottom-up components along plausible pathways to create a Reference Case (RC) scenario of global HFC-23 emissions projected through the next 25 years. This particular period is of interest in that its terminus (year 2035) is slightly beyond the concluding phase of the 2007 HCFC-related revisions to the Montreal Protocol, which bring global production and consumption of HCFC-22 for dispersive uses essentially to an end. It also coincides with the current term limits for renewable agreements for incineration of HFC-23 co-produced in the CDM projects.

Two variations on the RC scenario, those of Less Mitigation (LM) and Best Practices (BP), represent plausible upper and lower bounds to emission growth, respectively, corresponding to differing implementation of abatement measures. These three scenarios are offered as templates for policymakers to explore the potential implications of various current and future emission abatement measures on the HFC-23 radiative forcing component of climate change. Analogous to the intention behind the development of the emission scenarios for the upcoming IPCC 5th Assessment, the extensions presented here are intended to be "extrapolations based on simple rules, to avoid possible interpretation as true "scenarios"” (van Vuuren et al., 2009). Scenarios of global HFC23 emissions have been published previously (van Vuuren et al., 2010; Nakicenovic et al., 2000; Ottinger et al., 2006; McCulloch and Lindley, 2007). This current work differs from these predecessors in that it is based on existing policies only, including recent revisions to international agreements that significantly influence HFC-23 projections. The $\mathrm{RC}$ and derivative scenarios described here include various assumptions about how component strengths are constrained by historical trends, e.g., in United Nations Environment Programme (UNEP) data and CDM data, international regulatory schedules and programs, e.g., Montreal Protocol and CDM, and anticipated economic growth, e.g., GDP. Thus, the RC scenario and derivatives presented here do not include components of future HFC-23 emission intervention, which may be anticipated in the current policy climate and that have the potential to alter the course of HFC-23 emissions towards lower greenhouse gas emissions.

The following section begins with a description of the projected estimates for each of these components to derive the $\mathrm{RC}$ scenario, then concludes with the variations in abatement that produce the LM and BP scenarios. Note, in the discussion that follows, we use the terms "developed countries" and "developing countries" to mean Annex I and Non-Annex I countries under the Kyoto Protocol, respectively, and to mean Non-Article 5 and Article 5 countries under the Montreal Protocol, respectively. Note also that all data are plotted and tabulated as annual mean mid-year values. 


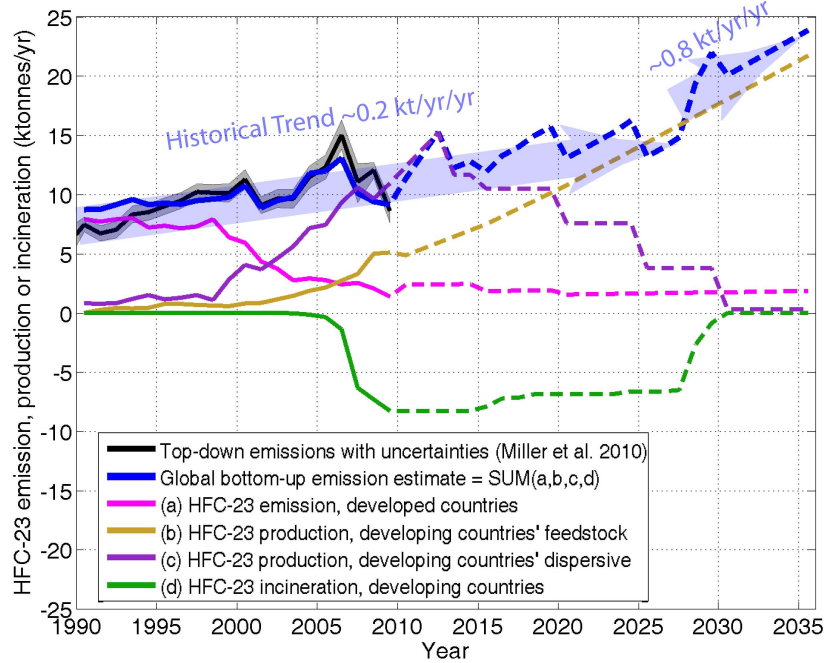

Fig. 1. Bottom-up HFC-23 emissions for developed countries (solid line a) (UNFCCC, 2010), and HFC-23 production and incineration for developing countries (solid lines b-d) (Miller et al., 2010), shown along with the bottom-up global HFC-23 emissions, which are the annual sums of each of these components. The bottom-up emissions, production and incineration are projected (dotted lines) to 2035 based on the "Reference Case" (RC) assumptions. The $\mathrm{RC}$ scenario suggests that the historical trend in emission growth of

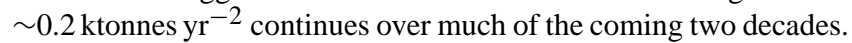
CDM project accreditation is scheduled to end by 2030, after which

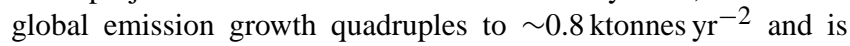
controlled indefinitely by the unrestricted production of HCFC-22 for feedstock use. Note that this increase occurs despite the complete phase out of HCFC-22 for dispersive uses by 2030. The topdown global emission estimates of Miller et al. (2010) (solid black line with uncertainties in gray) are shown as emission verification of the global bottom-up estimate.

\section{Emission model description}

\subsection{Developed countries' emissions}

Following a steep decline in HFC-23 emissions reported to the UNFCCC beginning about 1999, emissions from Annex I have remained fairly constant from 2004 through 2008 (Fig. 1). Emissions during this period appear to reflect a balance between declining production of HCFC-22 for dispersive uses, increasing production of HCFC-22 for feedstock use, implementation of process optimization, and voluntary incineration of HFC-23 at the production facilities. In projecting developed countries' HFC-23 emissions, it is assumed that this balance persists through 2035. Therefore, the Annex I 2008 emission value of 2.056 ktonnes HFC-23 is scaled by the ratio of each subsequent year's estimated HCFC-22 total production to the UNEP 2008 reported value of 292 ktonnes HCFC-22 (UNEP, 2010) to produce projected estimates for subsequent years.
For the RC scenario, a simplification is implied in our scaling of the 2008 developed countries' HFC-23 emissions by HCFC-22 annual production, namely that the amount of process optimization and incineration, relative to HCFC-22 production, remains constant through the next 25 years. Thus, we assume that these abatement measures that had such a dramatic effect on developed countries' emissions, starting in about 1999, had reached its potential for implementation by about 2004, an assumption that appears justified in view of the balance noted above between production and abatement.

In order to estimate the projected HCFC-22 total production for developed countries, we anticipated growth in dispersive and feedstock production separately. Dispersive production may be projected as the maximal annual values allowed under the 2007 revisions of the Montreal Protocol (UNEP, 2007). As defined under these revisions, production/consumption of the major HCFCs are phased out in steps as an ODP-weighted average, starting from a baseline of the 1989 ODP-weighted average of production and consumption of HCFCs plus $2.8 \%$ of the ODP-weighted average of production and consumption of CFCs. Based on UNEP production and consumption data (UNEP, 2010), the baseline HCFC production is 41777 ODP tonnes. If all of this baseline production were expressed as HCFC-22, then divided by the ODP value of 0.055 (Douglas et al., 2007) for HCFC22, this equates to 759582 tonnes HCFC-22. Rather than try to anticipate how future production/consumption of HCFC22 may be favored over that of the other HCFCs, we make the assumption that the 2005-2007 average ratio of HCFC22 production to that of the total HCFCs remains constant at 0.76 for the next 25 years. Thus each year's developed countries' dispersive production of HCFC-22 is projected as 759582 tonnes HCFC-22 multiplied by 0.76 and multiplied by the reduction fraction for that year given by the Montreal Protocol.

The remaining component of developed countries' HCFC22 total production, namely that for feedstock use, is extrapolated based on GDP growth of the developed world. Of three GDP projections offered by the EIA (2010), we chose to use the GDP Reference Case based on purchasing power parity. The EIA High Economic Growth and Low Economic Growth scenarios for the developed world offer $7.55 \%$ greater growth and $7.07 \%$ lesser growth, respectively, relative to the GDP Reference Case scenario integrated over the period 2005-2035. The sensitivity of feedstock growth to changes in GDP was determined from production data from UNEP (2010) and the GDP Reference Case projection from EIA from 2005-2007, then this sensitivity was applied to EIA projected GDP growth to produce projected feedstock production. The feedstock and dispersive production projected for developed and developing countries is shown in Fig. S1 (see SI text) and the projected HFC-23 emissions are shown in Fig. 1. 


\subsection{Developing countries' HFC-23 emissions from production of HCFC-22 for dispersive uses}

The 2007 revisions to the Montreal Protocol (UNEP, 2007) also advance the phase-out of HCFCs in the developing world. With a baseline defined as the average of 2009 and 2010 production and consumption of the major HCFCs, a freeze at this level is to take place in 2013. This is followed by a $10 \%$ reduction with respect to this baseline by 2015 , a $35 \%$ reduction by 2020 , a $67.5 \%$ reduction by 2025 and a $97.5 \%$ reduction by 2030 that is averaged over the next 10 years.

The average HCFC-22 dispersive production of 2009 2010 is used as a proxy for the developing world baseline. The UNEP 2009 developing countries' dispersive production value is 371 ktonnes. As an estimate of the 2010 to 2012 dispersive production, we assume the same growth rate observed for 2006-2007 (48.1 ktonnes $\mathrm{yr}^{-1}$ ) applies to growth of this period. Thus, in 2013, dispersive production of HCFC-22 is frozen at the mean 2009/2010 value of 395 ktonnes and all subsequent reductions are relative to this quantity.

We note that developed and developing countries may choose to reduce HCFC-22 production to a lower amount than the maximum production allowed under the Montreal Protocol. Therefore, the projections for HFC-23 production from dispersive use production of HCFC-22 are expected to be upper limits.

\subsection{Developing countries' HFC-23 emissions from production of HCFC-22 for feedstock}

For the developing world, we restrict our choice of GDP growth scenarios to that of China alone, where the dominant fraction of feedstock production is anticipated to occur (McCulloch and Lindley, 2007). We project feedstock production for China based on correlation with EIA's GDP Reference Case scenario. The EIA High Economic Growth and Low Economic Growth scenarios for China offer $7.64 \%$ greater growth and $7.00 \%$ lesser growth, respectively, relative to the GDP Reference Case scenario integrated over the period 2005-2035.

The feedstock and dispersive production projected for the developed and developing countries is shown in Fig. S1 (see SI text). It is worth noting that global production of HCFC22 for feedstock use is projected to surpass that for dispersive use by about 2015 , leaving the unrestricted production of feedstock as the dominant source of HFC-23 production thereafter.

\subsection{Developing countries' HFC-23/HCFC-22 co-production ratio}

In order to estimate the HFC-23 production from HCFC-22 production for dispersive and feedstock uses in developing countries, we multiply the HCFC-22 production projections by the average 2006-2009 value of the HFC-23/HCFC-22 co-production ratio, 0.0294, reported from the CDM project monitoring reports (Miller et al., 2010). To the extent that process optimization can be implemented in these facilities, it is noted that the resulting HFC-23 production from this source would tend toward the Best Practices scenario.

\subsection{Incineration of HFC-23 from HCFC-22 production in developing countries}

We project HFC-23 incineration in the developing world with the assumption that no additional capacity is installed beyond the 8 ktonnes $\mathrm{yr}^{-1}$ already demonstrated by 2009 within the facilities operating as projects under the CDM (Miller et al., 2010). During project registration, facilities opted for either a one-time fixed crediting period (at most ten years) or for a renewable crediting period (at most seven years) for up to a total of three periods. CDM projects in India opted for a fixed crediting period, while projects in China, South Korea, Mexico and Argentina opted for renewable seven-year periods. For the RC scenario it is assumed that the CDM projects that opted for seven-year approval periods are approved for a total of three consecutive terms, for a total of 21 years of abatement.

\subsection{Less Mitigation scenario}

The Less Mitigation (LM) scenario illustrates the change in HFC-23 emissions that may result if the twelve CDM projects that opted for seven-year crediting periods are not renewed after their first terms. Figures 2 and S2 (see SI text) illustrate the LM scenario.

Recent issues brought forward in the policy arena seem to remove some motivation for continuing incineration via the CDM projects, and it remains to be seen whether or not the CDM participants will choose to further incur the expense of mitigation. We note that the first seven-year crediting period for the South Korean plant in Ulsan ended in December 2009 , and as of June 2011, there has been no indication of project renewal. We also note the January 2011 recommendation of the European Commission that the EU cease the purchase of CERs derived from emission mitigation of HFC23 and $\mathrm{N}_{2} \mathrm{O}$ from adipic acid production after May 2013, implementation of which would remove the major market for these CERs. Thus, the present course may be tending toward the less mitigation scenario.

\subsection{Best Practices scenario}

Our Best Practices (BP) scenario suggests an HFC-23 emission projection that could result from additional abatement measures, namely global installation of full incineration capacity at all unmitigated HCFC-22 production facilities.

While a more wide spread implementation of process optimization alone would tend to reduce future emissions, the 


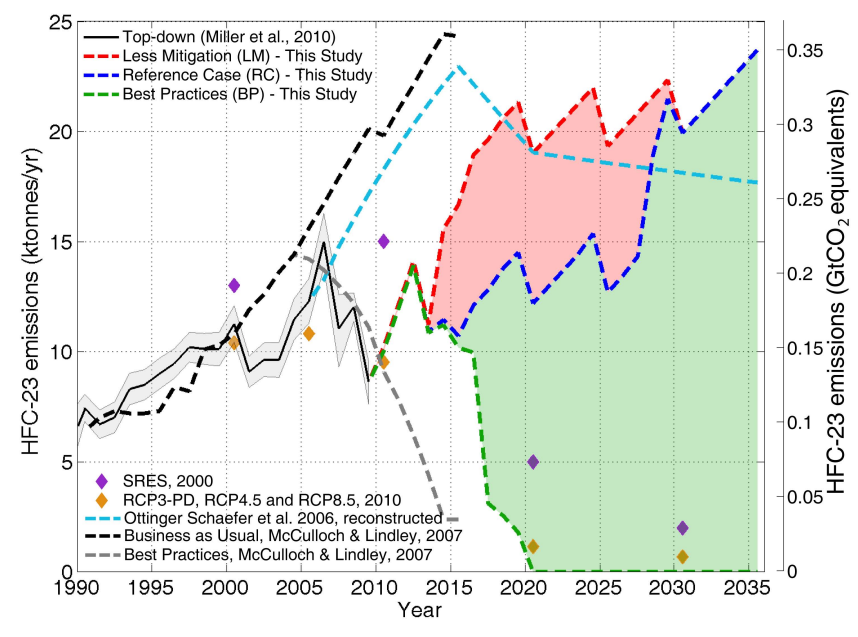

Fig. 2. HFC-23 global emission projections for the three scenarios presented in this paper, with the top-down emission estimates of Miller et al. (2010) as the starting point for projection. Shown for comparison are the SRES and RCP projections, and a reconstruction of the Ottinger Schaefer et al. (2006) projection based on the assumptions (Table S2) described in their paper. The Less Mitigation (LM) scenario depicts emissions if renewals of CDM projects are not approved. Implementation of additional incineration, a Best Practices (BP) scenario, could bring emissions to virtually zero after an initial startup lag. The RCP scenario most closely resembles our BP scenario.

effect may be limited to cutting developing countries' HFC23 production by less than $\sim 50 \%$. This assumes that the developed countries' have already implemented this abatement measure to nearly the fullest possible extent, and that facilities in developing countries can reduce the co-production ratio from the current average 0.0294 (Miller et al., 2010) to approach the lower limit of $\sim 0.014$ achieved by one of the largest facilities in the developed world (Rotherham, 2004). With an HFC-23 emission for developed countries anticipated at $\sim 1.55$ ktonnes in 2020 (see Table S1), reducing the HFC-23 production in developing countries by $50 \%$ (and assuming the amount of HFC-23 available for incineration by CDMs is also reduced $50 \%$ ) could reduce global emissions in 2020 to 7.3 ktonnes. This would be a $\sim 44 \%$ reduc-

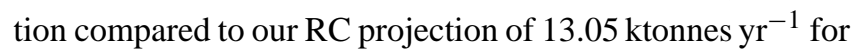
2020. Thus process optimization alone can make a significant emission reduction. However, arguments have been put forth that the amount of reduction possible through process optimization in the larger plants of the developed world may not be achievable in the smaller production facilities of the developing world (Lindley, 2004).

A more extensive reduction in emissions would be achieved by installation of full incineration capacity at all currently unmitigated HCFC-22 production facilities, in both the developed and developing world. We do not expect such wide scale mitigation to achieve zero "real world" emissions, for emissions are likely to continue from facilities while incinerators are down for repair and also from small banks of refrigeration sources. Estimation of the degree to which policymakers and industry might chose to mitigate such sources more thoroughly in the future is outside the aims of this paper.

Noting that it took approximately six years for the CDM projects to ramp up incineration capacity from zero to the

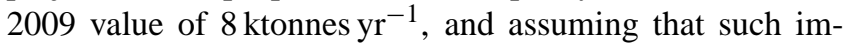
plementation would likely not commence until $\sim 2013$ or thereafter, the same startup period for full implementation of HFC-23 incineration globally could result in minimum emissions by 2020 . We use this scenario of globally installed HFC-23 thermal decomposition as our BP scenario, and suggest that it results in a lower limit for future HFC-23 emissions, and its atmospheric abundance and radiative forcing. Figures 2 and S3 (see SI text) illustrate the BP scenario (also see Table S1).

\section{Results}

\subsection{Projected emissions}

If controlled by the projected production and current abatement efforts, under the Reference Case scenario the HFC23 emission growth is projected to continue to follow the

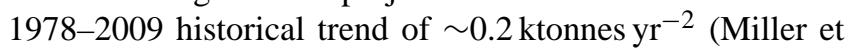
al., 2010) for most of the next two decades (Fig. 1). This trend would occur despite continuation of emission abatement measures that are already operational. These measures include the voluntary process optimization and HFC-23 incineration in developed countries, and incineration through the CDM projects in developing countries, all of which slightly decreased the slope of the trend temporarily during the 2000's. However, expiration of the accreditation periods for the CDM projects is slated for 2030, resulting in a sharp upturn in HFC-23 emission growth to almost 0.8 ktonnes $\mathrm{yr}^{-2}$ as incineration of $\sim 7 \mathrm{ktonnes} \mathrm{HFC-23/year}$ abruptly comes to an end while feedstock production continues to follow a projected rapidly rising GDP growth in China. The increase could occur much sooner, within the next five years, if the CDM projects are not renewed (i.e., the LM scenario) and other abatement measures do not take their place. At the other end of the spectrum, minimal emissions might result within the next decade with full implementation of HFC-23 incineration globally (i.e., the BP scenario).

\subsection{Projected atmospheric abundance and radiative forcing of HFC-23}

The atmospheric mole fractions that would be expected to result from the RC emission scenario, along with the upper and lower limits suggested by the LM and BP scenarios, are shown in Fig. 3. These projected abundances were determined by input of the emissions into a simple 3-box model (two lower troposphere boxes and one stratosphere box) and 


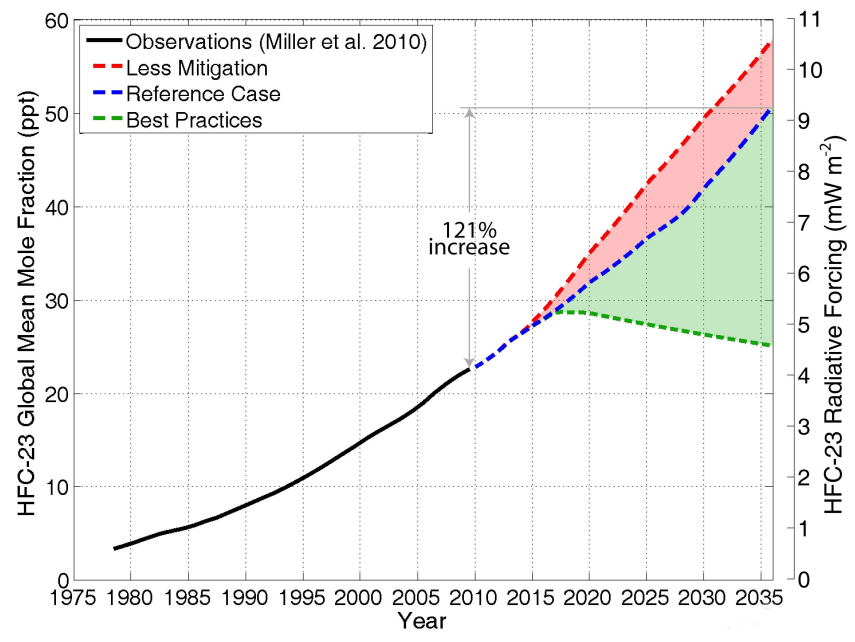

Fig. 3. Global HFC-23 atmospheric abundance (left axis) and radiative forcing (right axis) from observations (solid black line) (Miller et al., 2010) with projections based on the three emission scenarios of Fig. 2. The Reference Case scenario suggests that the nearly linear trend observed since $\sim 1995$ could continue well into the next two decades, followed by a sharp upturn with expiration of the accreditation periods of Clean Development Mechanism projects that incinerate the HFC-23 co-produced during HCFC-22 production in the developing world. Relative to the 2009 global atmospheric abundance of $22.8 \mathrm{ppt}$, the RC scenario suggests a $121 \%$ increase by 2035 , and the LM scenario suggests a $153 \%$ increase.

assuming tropospheric and stratospheric lifetimes of 260.3 and 2347 years (Naik et al., 2000), respectively.

The RC emissions produce an atmospheric trend well into the next two decades that is an extension of the almost linear growth rate observed from $\sim 1995$ to 2009 , then turns sharply upward by 2030 as the CDM projects go offline. Thus by year 2035, the atmospheric abundance of HFC-23 is projected at $50 \mathrm{ppt}$ (parts-per-trillion, $10^{-15}$ ), a $121 \%$ increase over the 2009 abundance. Extrapolating from the 2005 radiative forcing calculated for HFC-23 in AR4 of the IPCC (Solomon et al., 2007), by year 2035 the RC scenario yields a radiative forcing of $9 \mathrm{~mW} \mathrm{~m}^{-2}$.

The LM scenario suggests a $153 \%$ increase in HFC-23 atmospheric abundance to $57 \mathrm{ppt}$ over the same period. The BP scenario suggests a peak in HFC-23 mole fraction at $~ 27 \%$ greater abundance relative to 2009 to occur sometime late in the next decade, followed by a slow decrease driven by the long atmospheric lifetime of HFC-23.

\subsection{Comparison with other scenarios}

Greenhouse gas emission scenarios were developed to facilitate assessment of future climate change in the IPCC Third Assessment Report and in the upcoming IPCC Fifth Assessment Report. Here we compare the HFC-23 projections derived in this paper with those used in the IPCC climate scenarios to understand the implications of their respective assumptions. Fig. 2 compares these various emission scenarios.

For the IPCC Third Assessment Report, Working Group III created the Special Report on Emission Scenarios (SRES) (Nakicenovic et al., 2000). With input from community experts, emission projections were created to drive global circulation models to produce six climate change scenario groups from four qualitative storylines (A1, A2, B1 and B2). The same HFC-23 emission projection was used for all six of these scenarios. This projection shows a peak in HFC-23

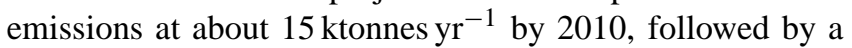

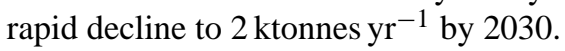

For the IPCC Fifth Assessment Report, a new set of emissions scenarios were developed by the research community. Four Representative Concentration Pathways (RCPs) specify radiative forcing through the end of the 21 st century (van Vuuren et al., 2009). Although the different RCPs individually address different radiative forcing pathways, the three scenarios (RCP3-PD, RCP4.5 and RCP8.5) that do include projections of HFC-23 emissions from HCFC-22 production use the same HFC-23 scenario. Van Vuuren et al. (2010) derived this HFC-23 emission scenario for the RCPs by adapting equations from Harnisch et al. (2009) into the IMAGE model. The original Harnisch et al. (2009) HFC-23 projection assumed "emissions from source distribution in 2020 are reduced by $20 \%$ every 5 years and set to zero after they have decreased below $5 \%$ of 2020 levels." Van Vuuren et al. (2010, and pers. comm.) compared the outcomes of these equations to results in Velders et al. (2009) and subsequently adjusted parameters for a better match. Although this resulted in a high baseline projection, it was subsequently reversed downward based on climate policy. The resulting HFC-23 emission scenario is in reasonable agreement with the top-down emission history of Miller et al. (2010) for years 2000-2010, then shows rapid decline to near zero emissions by 2030 .

The RCP HFC-23 emission scenario for 2020-2030 most closely resembles our BP scenario, which it should be recalled implemented thermal decomposition to all HCFC-22 producing facilities globally. It is not possible to tell from the description of the RCP scenario whether the dramatic reduction in emissions by 2020 is due to an anticipated decline in HCFC-22 production for feedstock or more widespread implementation of thermal decomposition, or a combination of these two factors. Given our expectation that feedstock growth follow the EIA GDP projections, then an average of 12 ktonnes per year incineration would be required during 2015-2025, in addition to what is already occurring in the developed world and through the CDMs, to achieve the low global emissions of the RCP for 2020. As feedstock continues to grow, the required additional incineration capacity would be effectively double the current capacity at more than

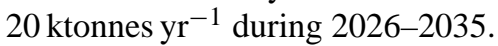

The earliest published projection of HFC-23 emissions to explicitly include assumptions about future HCFC-22 
production, the co-production ratio of HFC-23 to HCFC22 and abatement efforts was that of Ottinger Schaefer et al. (2006). Table S2 (see SI text) shows a comparison of the differences in assumptions between their study and that presented here, and Fig. 2 illustrates the differences in resulting emissions. Note that the Ottinger Schaefer et al. (2006) projection did not include the anticipated effect of HFC-23 incineration in the CDM projects, and that their study preceded the 2007 revisions to the Montreal Protocol that significantly accelerated the phase out of HCFC-22 production for dispersive uses.

\subsection{HFC-23 future emissions in comparison with the other major HFCs}

Velders et al. (2009) projected the growth of the major HFCs through 2050 and concluded that the global HFC emissions in 2050 could be equivalent to $9-19 \%\left(\mathrm{CO}_{2}\right.$-eq. basis) of projected global $\mathrm{CO}_{2}$ emissions in business-as-usual scenarios. These authors excluded HFC-23 from their calculations on the expectation that "future demand for HFC-23... is small compared to the other leading HFCs, especially past 2015." However, they did emphasize the significant potential for future emissions of HFC-23 to contribute to climate forcing due to its large GWP of 14800 , which is at least a factor of three greater than that of any one of the HFCs they considered. An extrapolation of the nearly linear segment 2030-2035 of our RC, which by that point is largely controlled by feedstock growth, suggests a 2050 emission rate of

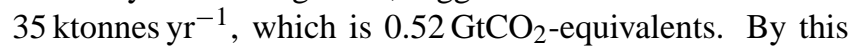
comparison, of the eight major HFCs in use today, HFC23 is projected to rank 4th highest in $\mathrm{CO}_{2}$-equivalents by 2050, just behind HFC-134a (Velders et al., 2009). If HFC23 had been included in the Velders et al. (2009) calculations, it would have amounted to 6-9\% of the total high and low estimates of $\mathrm{CO}_{2}$ equivalents from HFCs, respectively, and slightly raised the 9-19\% estimate of HFC contribution relative to SRES $\mathrm{CO}_{2}$ Reference Case scenarios. At a 2050 abundance of $78 \mathrm{ppt}$, HFC-23 would contribute $14 \mathrm{~mW} \mathrm{~m}^{-2}$ to radiative forcing, about 3-5\% of the total from HFCs (including HFC-23) for the Velders et al. (2009) high and low HFC scenarios.

\section{Conclusions}

In Miller et al. (2010), a global bottom-up HFC-23 emission history for 1990-2008 was constructed from production, emission and thermal decomposition data. Here, these same bottom-up component terms are projected to year 2035 to produce a global bottom-up HFC-23 emission scenario that offers insight into how the atmosphere might change with and without additional efforts to constrain HFC-23 emissions. This Reference Case (RC) scenario relies on the existing framework of international agreements for phase out of
HCFC production and on existing HFC-23 emission abatement efforts in both developed and developing countries, and it anticipates growth in the source strengths based on projected GDP growth and restrictions of the Montreal Protocol.

Assuming this RC scenario, global HFC-23 emission growth is projected to continue to follow the 1978-2009 his-

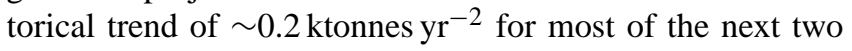
decades. This trend would result despite anticipated continuation of emission abatement measures that are already operational. By year 2030 the CDM accreditation periods are slated to expire, resulting in a sharp upturn in HFC-23 emission growth to $\sim 0.8 \mathrm{ktonnes} \mathrm{yr}^{-2}$. This increased emission rate results as CDM incineration of $\sim 7$ ktonnes HFC-23/year abruptly comes to an end while feedstock production continues to follow a projected rapidly rising GDP growth in China. Under this RC scenario, the resulting atmospheric abundance of HFC-23 is expected to be 50 ppt by 2035 , an increase of $121 \%$ over the 2009 observed mole fraction.

A variation on the $\mathrm{RC}$ scenario, that of a Less Mitigation (LM) scenario, suggests that the upturn in HFC-23 emission growth could occur as early as 2014 if the CDM projects that are eligible for renewal for a second and third term are not approved. The resulting atmospheric abundance is anticipated to increase $153 \%$ by year 2035 relative to that of 2009 .

A second variation of the RC, that of a Best Practices (BP) scenario, anticipates the increasing implementation of thermal decomposition of HFC-23 needed if emissions are to be decreased to and maintained at minimal levels in the coming decades. By year 2035, this could require about

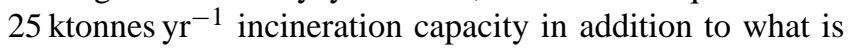
currently in place in the developed world. Such a scenario could limit the atmospheric abundance of HFC-23 to 25\% greater than the 2009 observed mole fraction, followed by a slow decay over the coming decades due to the long atmospheric lifetime.

Global production of HCFC-22 for feedstock use is anticipated to surpass production for dispersive uses by about 2015 as the 2007 revisions to the Montreal Protocol rapidly phase out the latter. After 2030 in the RC scenario, when the Montreal Protocol HCFC phase out is complete and after the CDM projects have all expired, the growth in HFC-23 production will be controlled by feedstock production and process optimization. Thus, in the long term, the controlling factor determining whether the recent decline in HFC23 emissions reported by Miller et al. (2010) continues or there is a resurgence in emission growth may be the extent to which incineration can keep pace to counteract growth of this unrestricted feedstock production.

A comparison of the $\mathrm{RC}$ and derivative scenarios with several previously published HFC-23 emission scenarios suggests that each of the latter implicitly expect HCFC-22 production to be a small fraction of that modeled here and/or include some anticipation of extensive, additional HFC-23 emission abatement significantly beyond that which currently exists. For example, in the decade 2026-2035, the RCP 
HFC-23 emission projection would require additional HFC23 thermal decomposition that is effectively double the current capacity in place in the developed and developing world,

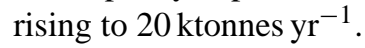

The recent study of Velders et al. (2009) showed the significant contribution by year 2050 of HFC emissions to global radiative forcing, relative to SRES Reference Case $\mathrm{CO}_{2}$ emission scenarios. However, they excluded HFC-23 from those calculations. The RC and LM scenarios of the current study suggest that by the year 2050 HFC-23 emissions could account for 0.52 equivalent $\mathrm{GtCO}_{2}$. When this is combined with the contribution of other HFCs from Velders et al. (2009) HFC-23 emissions amount to $6-9 \%$ of total $\mathrm{CO}_{2}-$ equivalents from all HFC emissions, making HFC-23 emissions a non-negligible component of climate change projections.

\section{Supplementary material related to this article is available online at: http://www.atmos-chem-phys.net/11/13259/2011/ acp-11-13259-2011-supplement.pdf.}

Acknowledgements. We acknowledge the support of S. Montzka for this work. We thank P. Fraser for beneficial discussions. The authors also wish to thank the RCP community, particularly D. van Vuuren, for helpful information regarding RCP development.

Edited by: N. M. Donahue

\section{References}

Douglas, A. R., Forster, P. M. D., Hauglustaine, D. A., Isaksen, I. S. A., Kuijpers, L. J. M., McCulloch, A., and Wallington, T. J.: Halocarbon scenarios, ozone depletion potentials, and global warming potentials, in: Scientific Assessment of Ozone Depletion: 2006, Global Ozone Research and Monitoring Project report no. 50, (World Meteorological Organization, Geneva, Switzerland) 8.1-8.39, 2007.

EIA (2010) International Energy Outlook 2010, Reference Case Projections, Appendix A (US Energy Information Administration) available online at: http://www.eia.doe.gov/oiaf/ieo/pdf/ ieorefcase.pdf, last access: December 2011, 2010.

EPA, (2006) EPA 430-R-06-005, "Global Mitigation of Non-CO2 Greenhouse Gases", available at http://www.epa.gov/climatechange/economics/downloads/ GlobalMitigationFullReport.pdf, last access: December 2011, 2006.

Forster, P., Ramaswamy, V., Artaxo, P., Berntsen, T., Betts, R., Fahey, D. W., Haywood, J., Lean, J., Lowe, D. C., Myhre, G., Nganga, J., Prinn, R., Raga, G., Schulz, M., and Van Dorland, R.: Changes in Atmospheric Constituents and in Radiative Forcing, in: Climate Change 2007: The Physical Science Basis. Contribution of Working Group I to the Fourth Assessment Report of the Intergovernmental Panel on Climate Change, edited by: Solomon, S., Qin, D., Manning, M., Chen, Z., Marquis, M., Av- eryt, K. B., Tignor, M., and Miller, H. L., Cambridge University Press, Cambridge, UK and New York, NY, USA, 2007.

Harnisch, J., Klaus, S., Wartmann, S., and Rhiemeier, J.-M.: Documentation Report - Development of F-Gas module for timer model, (Ecofys for the Netherlands Environmental Assessment Agency MNP), 2009.

Lindley, A.: Response to DuPont letter to CDM Methodology Panel concerning AM0001, available online at http://cdm.unfccc.int/public_inputs/inputam0001/ CommentAM0001_IneosFluor_LettertoMP_05July2004.pdf, last access: December 2011, 2004.

McCulloch, A. and Lindley, A. A.: Global emissions of HFC23 estimated to year 2015, Atmos. Environ., 41, 1560-1566, doi:10.1016/j.atmosenv.2006.02.021, 2007.

Miller, B. R., Rigby, M., Kuijpers, L. J. M., Krummel, P. B., Steele, L. P., Leist, M., Fraser, P. J., McCulloch, A., Harth, C., Salameh, P., Mühle, J., Weiss, R. F., Prinn, R. G., Wang, R. H. J., O’Doherty, S., Greally, B. R., and Simmonds, P. G.: HFC-23 (CHF3) emission trend response to HCFC-22 (CHClF2) production and recent HFC-23 emission abatement measures, Atmos. Chem. Phys., 10, 7875-7890, doi:10.5194/acp-10-7875-2010, 2010.

Naik, V., Jain, A. K., Patten, K. O., and Wuebbles, D. J.: Consistent sets of atmospheric lifetimes and radiative forcings on climate for CFC replacements: HCFCs and HFCs, J. Geophys. Res., 105, 6903-6914, 2000.

Nakicenovic, N. and Swart, R., Editors: Special Report on Emission Scenarios, Working Group III (IPCC), available online at http:// www.grida.no/publications/other/ipcc_sr, last access: December 2011, 2000.

Ottinger Schaefer, D., Godwin, D., and Harnisch, J.: Estimating future emissions and potential reductions of HFCs, PFCs, and SF6, Energy J. Special Issue, 3, 63-88, 2006.

Rotherham, D.: Greenhouse Gas Emission Reduction Verification Audit for Dupont's Louisville Works Freon®22 Plant, Final Report (ICF Consulting, Toronto, Canada) available online at: http://cdm.unfccc.int/public_inputs/inputam0001/Letter Dupont_Annex2_03June04.pdf, last access: December 2011, 2004.

Solomon, S., Qin, D., Manning, M., Chen, Z., Marquis, M., Averyt, K. B., Tignor, M., and Miller, H. L.: Climate Change 2007: The Physical Basis, Contribution of Working Group I to the Fourth Assessment Report of the Intergovernmental Panel on Climate Change, Cambridge University Press, Cambridge, UK, 2007.

UNEP: Report of the 9th Meeting of the Parties to the Montreal Protocol on Substances that Deplete the Ozone Layer, UNEP/OzL. Pro. 19/7, (United Nations Environment Programme, Nairobi, Kenya), 2007.

UNEP: Production and Feedstock Production data for HCFC-22, 1989-2008, as reported under Article 7 of the Montreal Protocol (UNEP Nairobi), March 2010.

UNFCCC: data in the Common Reporting Format, available online at: http://unfccc.int/di/DetailedByParty.do, last access: December 2011, 2010.

UNFCCC: CDM Executive Board, Annex 11 - Executive summary of the Meth Panel report on HFC23 issues (AM0001), November 2010, available online at: http://cdm.unfccc.int/Reference/Notes/ meth_note02.pdf, last access: December 2011, 2010. 
van Vuuren, D., Riahi, K., Smith, S., Meinshausen, M., Mastrandrea, M., and Moss, R.,: RCP Extension White Paper, available online at: http://cmip-pcmdi.llnl.gov/cmip5/docs/ DraftRCPExtension_WhitePaper_26Ju109.pdf, 2009.

van Vuuren, D. P., Stehfest, E., den Elzen, M. G. J., van Vliet, J., and Isaac, M.: Exploring IMAGE model scenarios that keep greenhouse gas radiative forcing below 3 $\mathrm{W} / \mathrm{m}^{2}$ in 2100 , Energy Economics, document 32, 1105-1120, doi:10.1016/j.eneco.2010.03.001, last access: December 2011, 2010 .
Velders, G. J. M., Fahey, D. W., Daniel, J. S., McFarland, M., and Andersen, S. O.: The large contribution of projected HFC emissions to future climate forcing, Proc. Nat. Acad. Sci., 106, 10949-10954, 2009.

WMO (World Meteorological Organization), Scientific Assessment of Ozone Depletion: 2010, Global Ozone Research and Monitoring Project-Report No. 52, 516 pp., Geneva, Switzerland, 2011. 